\title{
Experimental Investigation of Solidification and Remelting over Cryocooled Sphere Using Different Concentrations of Brine Solution as Phase Change Material
}

\author{
Manoj Kumar' ${ }^{1}$ Rasmikanti Biswal ${ }^{1}$, Amitesh Kumar ${ }^{2 *}$ \\ ${ }^{1}$ Department of Mechanical Engineering, NIT, Rourkela, Odisha 769008, India \\ ${ }^{2}$ Department of Mechanical Engineering, IIT, Varanasi, U.P. 221005, India
}

Corresponding Author Email: kumar.amitesh@gmail.com

https://doi.org/10.18280/ijht.370103

Received: 7 January 2018

Accepted: 24 October 2018

Keywords:
brine solution, solid sphere,
$\begin{aligned} & \text { solidification, melting, } \\ & \text { convection }\end{aligned}$

\begin{abstract}
In this investigation, the solidification, phase change, melting and heat transfer phenomenon from the outer surface of the solid sphere (stainless steel) having different dimensions were analyzed. Liquid nitrogen has been used to cool the sphere at a cryogenic temperature up to $192{ }^{\circ} \mathrm{C}$, then dipped the sphere inside a phase change material (brine solution $\mathrm{NaCl}-\mathrm{H}_{2} \mathrm{O}$ of different concentration at ambient temperature) and study the solidification and melting phenomenon. The thickness of ice formation at the outer surface of a sphere is measured for different concentration of brine solution. Variation of ice thickness is also measured for different concentration at different time intervals. It is interesting to note that the time duration of solidification of ice and melting is depending upon the concentration of the solution. Melting behavior of ice is discussed for different concentration.
\end{abstract}

\section{INTRODUCTION}

Phase change materials (PCM's) are heavily used nowadays for thermal energy storage (TES) purpose. A lot of research work has been going on this part due to energy crisis faced by the world to save the energy. Total eighty-eight PCM's are available to us, out of which 40 is used commercially [1]. Spheres are very useful for energy storage purpose. The experiment is performed to discuss the behavior of chrome steel at a cryogenic temperature as well as inside the phase change material (brine solution at different concentration). PCM's are generally used for storing of solar energy, cooling of a turbine blade, heating/cooling of a building, liquid storage tank, cooling of nuclear fuel elements industrial process cooling etc. Theoretical and experimental work regarding brine solution/ice interface is a matter of interest due to its presence in natural, environmental, biological, geology, oceanography, metallurgical, physical, crystal growth and chemical phenomenon [2]. J. Stefan [3] has proposed the theory related to heat transfer phenomenon with phase change problems in 1889. Generally, inorganic salts dissolve endothermal and their solubility increases with increase in temperature. If the salt solution is saturated in a temperature range than the heat absorbing capacity per unit volume has been increased as compared to water and $\mathrm{NaCl}$ separately. Choi and Viskanta [4] provide the experimental results, which validate that supercooling phenomenon depends on the initial concentration of salt. When the salt concentration is $5 \%$, supercooling occurs at an early stage only, whereas for $15 \%$ it occurs throughout the process.

Matsumoto et al. [5] explained the solidification on a vertical wall of a rectangular cell. They plot the graphical result for permeability and volume fraction of liquid in the mushy region, which depends on initial salt concentration. If we decrease the initial concentration, natural convection and rate of concentration rise in liquid region decreases but the movement of freezing front will be increased. Okawa et al. [6] discussed between surface area and degree of supercooling and freezing of silver iodide in water. Freezing temperature is a function of the area exposed to the water, cooling condition and wettability.

Vrbka and Jungwirth [7] performed an experiment, compare their result with the help of molecular dynamic simulation and provides a molecular brine rejection process. They explained the rejection of salt ion on the ice front slowed the ice formation. Lucas et al. simulated numerically for porous medium freezing in aqueous concentration salt solution, changes in salt concentration, and ice fraction and temperature field with respect to time. Initially, the salt diffusion process is too fast to prevent freezing at the interface. They also provide a numerical solution by using Fick's law and compare the result with the experimental solution [8].

Valenza and Scherer [9] performed the experiment in a brine solution, which explained that volume concentration depends on the initial concentration of salt solution and it is a function of temperature. Raoult's law suggested that the vapor pressure of a dilute salt solution is less than that of pure water. According to Wilson and Haymet, Reynolds freezing potential indicates that initial result of freezing potential of a probe into DC electrical resistance is a function of salt concentration. They compared the result graphically between WorkmenReynold effect or freezing potential (WRFP) and growth of polycrystalline ice with time [10]. Frederking and Clark discuss the cooling phenomenon of a hot sphere in Liquid Nitrogen.

They assume constant physical properties, isothermal wall condition, negligible dissipation, no radiation, smooth surfaces, incompressible fluid and surface of the sphere is sufficiently large to create a vapor film of nitrogen, which moves upward. In this case, a vapor-liquid interface is not too 
smooth but for the same experiment performed on the horizontal cylinder shows, roughly $10 \%$ increase in smoothness. They derive the relation for heat transfer and shows that, $\mathrm{Nu}=\mathrm{f}(\Delta \mathrm{T}, \mu, \rho, \mathrm{Pr}, \mathrm{Gr}, \mathrm{h}, \mathrm{Cp})$ [11]. Their work is continued by David E. Daney [12] who performed the experiment to watch the natural convection of liquid deuterium, hydrogen (l) and nitrogen (l) within a sphere, hemisphere, horizontal cylinder, vertical cylinder. $\mathrm{He}$ compared the result with internal heat generation with quasisteady state. The result obtains corresponding to natural convection and variation of $\mathrm{Nu}$ and $\mathrm{Ra}$ is $\mathrm{Nu}=0.104 \mathrm{Ra} 0.352$. The experiment had great importance in cooling of the turbine blade, buildings, nuclear fuel element, and industrial process cooling. Four years later, Hilal and Boom [13] performed an experiment with a sphere suspended through a nylon rod inside a partially filled liquid helium cylindrical bath and observe the free convection phenomenon. They assume $\mathrm{Cp}, \beta, \mu$ and $\mathrm{k}$ as a constant and find the relation between $\mathrm{Nu}=\mathrm{f}(\Delta \mathrm{T}, \Delta \rho, \operatorname{Pr}$, $\mathrm{Gr})$. The main feature of this experiment is the boiling like phenomenon has occurred near the critical point which is not obtained previously. Further work was performed on a cylinder, for example, Merkin et al. [14] studied the combined convection boundary layer on a horizontal circular cylinder in a stream flowing vertically upward is studied in both the cases of a heated and cold cylinder. He observed that heating of a cylinder delayed the separation of boundary layer whereas Cooling cylinder brings the separation point nearer to the lower stagnation point but for an extremely cold cylinder, there will not be a boundary layer on the cylinder. Later on, the work was performed by considering the buoyancy effect by K. H. Bang [15] considered the buoyancy effect in vapor momentum equation for forced convection film boiling over a sphere for liquid and vapor flows, which was not used before. Buoyancy term was neglected if liquid velocity is high but for medium velocity (up to $7 \mathrm{~m} / \mathrm{s}$ ); the velocity of a liquid-vapor interface is larger or smaller than the free stream liquid velocity depends on the magnitude of buoyancy. He found that for high liquid sub-cooling, the film thickness is about 10 microns. As sub-cooling of liquid increases, the film thickness becomes thinner. The study is continued on plates, for example, Rahli et al. [16] performed the experiment for evaluation of temperature over space and time for three zones. (a) Liquid (b) Two-phase (c) Superheated vapor zone. They took bronze plates, which were heated by electrical resistance in a ceramic block. Copper plates were inserted between the bronze plate and heater for equivalent temperature difference and enclosed it in a closed asbestos. Liquid (n-pentane) flowed through the porous media from bottom to top vertically. They discussed the behavior of single-phase liquid and vapor zones (having high amplitude profile) and two-phase zone (having a relatively flat profile). When the phase change occurs, determine the pressure and velocity field's boundaries and their spatial and temporal evolution of temperature profile. They also obtained the hydrodynamic pressure of liquid and vapor zone by Darcy's law. Later on, Kolev [17] took the reference of Frederking et al. and perform the same for mixed convection film boiling over vertical wall and result was compared with spheres. Liquid (water) was flowing around a vertical plate $\&$ hot sphere due to which mixed convection is going on. Mass, momentum energy and Achenbach correlation $(\mathrm{Nu}=3.71+0.402(\mathrm{Gr} \times \mathrm{Pr}) 0.5$ was used to find heat transfer coefficient. He found that for a vertical surface with $10 \%$ accuracy over spherical one.

Miller et al. [18] performed a similar experiment on a copper plate. They took a perforated copper plate immersed it in liquid nitrogen and study the film boiling near the plate. Heat transferred to liquid nitrogen due to which bubbles of nitrogen gas were formed. They also take the effect of surface tension at terrestrial gravity. In the twenty-first century, the experiment was continued on a sphere by taking different phase change materials, for example, Ettouney et al. performed the experiment over a metal screen/sphere. Steel spheres and thermocouples were set at different angles and different heights, packed in a phase change material (paraffin wax) tube. The heat transfer fluid (water) was passed at different temperature and at a different flow rate over the metal screen. They found that $\mathrm{Nu}=\mathrm{f}(\mathrm{Ra}, \mathrm{St}, \mathrm{Fo})$, heat transfer coefficient in the PCM and HTF, the temperature profile of thermocouple, a radial temperature profile of PCM at a different height, axial temperature profile at various HTF temperature by using data acquisition unit [19]. H. Koizumi performed the experiment to show the melting pattern and heat transfer phenomenon with n-octadecane as solid PCM, which was filled in a copper sphere, heated by air with or without copper plate inserted in it. He obtained three flow patterns. (a) Chaotic flow obtained at $\mathrm{Re}=120$ and $\mathrm{Gr} / \mathrm{Re}^{2}=23$. (b) Two dimensional steady separated flow for $\mathrm{Re}=330$ and $\mathrm{Gr} / \mathrm{Re}^{2}=3$. (c) Three dimensional unsteady separated flow for $\mathrm{Re}=1800$ and $\mathrm{Gr} / \mathrm{Re}^{2}=0.1$. He found that latent heat storage rate was increased by inserting a copper plate inside the capsule [20]. Later on, a similar experiment was performed by A. Bermansour [21] who preferred Wood et al. and performed an experiment with hollow sphere filled with paraffin wax as a phase change material to use it as a thermal energy storage medium in a water system. However, they used airflow at a variable mass flow rate as a working fluid flow through the packed bed of sphere filled with paraffin wax. They observed that air and PCM temperature had been increased initially at the same rate but at the later stage the air temperature exceeded and the bed was fully charged. Theoretical and numerical results conclude that accurate prediction of temperature distribution within the bed during charging and discharging is possible. In 2008, two similar works were performed by F.L.Tan [22]. He investigated the melting of PCM inside a glass sphere for constrained and unconstrained melting. In constrained melting solid PCM, (n-octadecane) was restrained from sinking to the bottom of the sphere and produce waviness profile due to heat conduction at the inner wall is dominated because of increase in heating time. In unconstrained melting solid PCM sinks to the bottom due to gravity and buoyancy force, a high melting rate at the bottom region and initial time (low heat). He filled the PCM inside the sphere; flow hot water overheats with the help of experimental set-up due to temperature difference the PCM melts and observe the melting phenomenon with respect to a different time interval. He used a spherical container as a thermal energy storage to store PCM due to low volume to heat transfer surface area ratio. He found the melting characteristics of superimposed phase front $\mathrm{V} / \mathrm{s}$ time for constrained and unconstrained melting.

Tan et al. [23] performed the similar type experiment with constrained melting by using paraffin wax n-octadecane as 'PCM' filled in a glass sphere. Eight thermocouples (k-type) was used at a different location to find the temperature at various location and compare the result with the outcome of CFD. They draw the graph for temperature versus time for constrained melting. They found that at the top region melting was faster than the bottom region (waviness profile) whereas CFD result and experimental results were similar. In the same 
year, the studies were going on by Jouhara and Axcell [24]. The transient film boiling experiment was performed with different coolant velocities on two sphere, cylinder, and plates of different shapes. The objects were heated and then dipped in cooled water. They observed that turbulent and ripped type liquid/vapor interface was obtained for cylinders and planes whereas smooth interface occurred specifically on sphere only. There was no effect of water velocity except for very small cylinders. The vapor film was collapsed in a different manner at different temperature with explosive, progressive \& explosive-progressive manner. Kousksou et al. performed the work on crystallization. They assumed two cases for hydrodynamic and heat transfer characteristics in a horizontal circular tube for a suspension flow with micro size PCM particles. First, melting of phase change suspension with the n-Eicosane particle. Second, water as PCM to count the effect of the supercooling phenomenon. They found time evolution of suspension temperature for a different radial position with or without supercooling and crystallization. They compared the results to others with the help of histograms for wall heat flux v/s wall temperature, Stefan number V/s wall temperature, the temperature of crystallization and number of particles crystallized [25].

In 2012, A.R.M. Kasim performed an experiment on an isothermally heated horizontal cylinder by considering it as a solid sphere which is immersed in a viscous and incompressible fluid at ambient temperature. The surface of a sphere was subjected to Newtonian heating. The effect of Prandtl number on velocity, temperature, thermal boundary layer, skin friction, thermal diffusivity was solved by Kellerbox method and discussed through graphical representation [26]. Later the studies continued on nano-enhanced phase change material for the different cross section by Hosseinizadeh et al. [27]. They worked for the enhancement of melting rate of nano-enhanced phase change material (NEPCM) with respect to conventional PCM due to increase in thermal conductivity and by reducing the latent heat of fusion, they performed the experiment. They filled the NEPCM (Rubitherm $\mathrm{GmbH}$ ) inside a copper sphere (85\%) and coated the sphere with plexiglass. By providing the temperature difference, they observed the symmetry of phase change phenomenon of NEPCM inside the sphere with respect to time. Matin et al. [28] numerically studied the twodimensional steady-state natural convection of nonNewtonian power-law fluid (pseudo-plastic and dilatants) between two eccentric horizontal square duct (inner duct is hot and the outer duct is cold) by using second-order upwind scheme and algorithm. The effect of power law index (n), Ra, aspect ratio, eccentricity and Prandtl number were studied on heat and fluid flows. They found if ' $n$ ' was inversely proportional to 'Nu'. 'Pr' had no effect on heat transfer rate except for some cases. Convection transport phenomenon is dominant for pseudoplastic fluid compared to dilatant fluid. They also draw the streamlines and isotherms by taking different parameter into consideration. Tham and Nazar [29] studied steady laminar mixed convection boundary layer flow about the isothermal solid sphere in a porous medium filled with nanofluid for forward and retard flow. Mixed convection parameter ' $\lambda$ ', Nano-particles $\left(\mathrm{Al}_{2} \mathrm{O}_{3}, \mathrm{Cu}, \mathrm{TiO}_{2}\right)$ and Nanoparticle volume fraction ' $\varphi$ ' are measured for discussions about the effect on skin friction coefficient, boundary layer separation etc. Keller-box method was used to solve the problem.

Oro et al. [30] focused on thermal energy storage techniques, encapsulation, heat transfer enhancement and effect of storage with phase change material for cold applications. The main objective was to study various PCM for a different application. They used solid-liquid phase change to store a large quantity of energy. Zhao et al. [31] have continued similar work. They use NaNo3 and n-octadecane as encapsulated phase change material 'EPCM' in a stainless steel cylinder for hightemperature energy storage unit which was used in solar and nuclear power plant application. They used enthalpy porosity method and front tracking method for solving the problem with experiment and ANSYS Fluent. They found the isotherms and streamline by using the two techniques for both EPCM. They used a horizontal cylinder for cross-flow heat transfer (HTF) and vertical cylinder for axial flow of HTF at a higher temperature.

Kiani et al. [32] performed the experiment on a copper sphere cooled in an ethylene glycol-water mixture (1:1) under the influence of ultrasonic radiation. They showed that ultrasonic irradiation increases the heat transfer rate and ' $\mathrm{Nu}$ ' between the cooling object and the body, which was dipped in it. They also suggested the effect of 'Re' \& 'Pr' on 'Nu'. Vanderlaan and Sciver studied about the helium (He II) contained in a bed of polyethylene spheres of uniform size which was randomly packed in a stainless steel channel. Three flow regimes are studied. (a) The laminar regime in which temperature gradient is directly proportional to heat flux. (b) The turbulent regime in which temperature gradient was directly proportional to heat rate. (c) Transitional regime occurred by combining the laminar and turbulent regime. London's equation was used for laminar He II flow to relate the pressure and temperature gradient. Later Solomon and Velraj performed an excellent work for heat exchanger on fins. They used eight longitudinal copper fins placed at different heights on the outer surface of a copper tube and study the increase in heat transfer performance of PCM (paraffin) used in the free cooling application. Fins were very useful in sensible cooling of liquid PCM. They utilized the performance of fin during solidification at a different height. The effect of a fin on subcooling and sensible cooling, inlet air velocity, and heat transfer fluid was explained.

Hashemi et al. [33] perform some experiments on sedimentation phenomenon at a different temperature. They use Lattice-Boltzmann method to simulate fluid particles interaction. They take 30 hot spheres which are dropped inside a box filled with relatively low-temperature Si-oil box and examine the sedimentation phenomenon at a varied temperature at different times, Re, Gr \& Pr. They depict that the density ratio and temperature difference are the factors which define the moving direction of the particle. Amin et al. [34] also compare later experimental work with CFD simulation. The CFD simulation of heat transfer fluid (HTF) flowing past a sphere with phase change material (water as PCM) and comparison were made against the measured and modeled temperature at the center of a sphere. The CFD model correlates with the freezing phenomenon shows that the buoyancy effect is negligible. When melting test was compared to the CFD model, the effective thermal conductivity of liquid layer in the sphere could be determined over the melting process.

In this paper, the solidification and melting phenomenon of different concentration of brine solution over different diameters of the cryocooled solid sphere have been reported. The ice thickness at various locations is compared for different solids. The melting pattern of ice is also discussed. 


\section{EXPERIMENTAL SETUP}

\subsection{Material and methods}

Schematic diagram of the experimental setup is shown in the Figure 1. Polymethyl methacrylic (PMMA) sheet having 5 $\mathrm{mm}$ thickness had been used to make a container having inside dimensions $301 \mathrm{~mm}$ width, $302 \mathrm{~mm}$ length and $301 \mathrm{~mm}$ height and the top surface has been open to atmosphere. The container was made by PMMA due to a better visible assessment of solidification as well as melting phenomenon and to capture the images of all processes. PMMA sheet is cut with the help of hacksaw in the specified dimensions then file its surfaces and assemble it with the help of silicone gel and chloroform. A thin layer of silicone gel is glued to the edges of acrylic pieces, assemble it and left for 10 to 12 hours for gaining the strength. After that minute holes are filled with acrylic powder and chloroform was sprayed on it with the help of a syringe. Due to the corrosive behavior of chloroform, it is carefully sprayed at the place where the acrylic powder was used to fill the minute holes. The volume of the container is sufficiently large to use it as a sink.

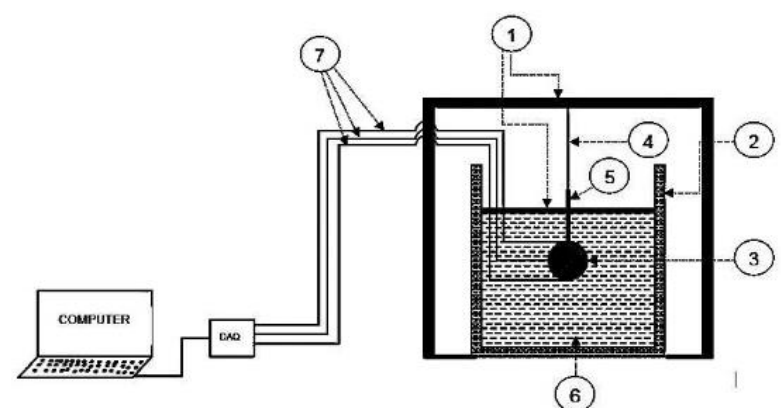

Figure 1. Schematic diagram of experimental setup (1.Wooden structure 2.Acrylic container 3. Stainless steel sphere 4. High modulus vectran fibre wire 5.Plastic pipe 6 . Brine solution 7. K-type thermocouple wire)

\subsection{Temperature measurement}

According to "American Society for Testing and Materials" (ASTM 230-87) standard, K-type thermocouple (chromel and alumel) had been used to measure the temperature at different locations (Top $\left(\theta=0^{\circ}\right)$, side $\left(\theta=90^{\circ}\right)$ and bottom $\left.\left(\theta=180^{\circ}\right)\right)$ of the sphere. The bead has been made with the help of soldering iron, flux, and lead-tin alloy. The bead has been made in a spherical shape having $1 \mathrm{~mm}$ to $1.5 \mathrm{~mm}$ diameter, clip it with high-density polyethylene net in which sphere was hanged. Since, the sphere is hanged inside the high-density polyethylene net, due to a load of the sphere; thermocouple, which is attached to the net, holds its position. In this manner, the surface of the sphere came in contact with the thermocouple bead. The thermocouple wire at the top is attached by passing it through small diameter plastic pipe, which is used for stability of the sphere. After placing the thermocouple at its specified position, attach it to Data Acquisition System (DAQ system) which convert the thermal signal into the emf signal. The standard tolerance of K-type thermocouple wire lies within $\pm 0.75 \%$. Lab View and National Instrument software were used to measure the temperature with respect to time by "Data Acquisition System" (DAQ). Now finally, DAQ is connected through a data cable to the computer. By connecting it to the lab view software, we were able to measure the temperature versus time graph on the computer screen.

\subsection{Experimental procedure}

The assumptions considered for performing the experiment that dimensions of stainless steel sphere remain constant as well as properties like thermal conductivity, the density of the material and surface roughness of the sphere are not changed when it was immersed inside the liquid nitrogen. The sphere removed from liquid nitrogen when the steady-state temperature is reached, dipped it into the brine solution. It is assumed that the process had been completed within a fraction of second but practically it will take 5 to 7 seconds.

The experiment was performed on the solid sphere of same material (stainless steel) having different diameters of 4.14 $\mathrm{mm}, 3.18 \mathrm{~mm}$ and $2.70 \mathrm{~mm}$ respectively. The sphere was encased in high-density polyethylene net and tightened it with cotton thread. High modulus vectran fiber wire was attached to the cotton thread for holding purpose. It is passed through a plastic pipe for stability and to avoid forced convection when it was immersed in the brine solution. Now, a k-type thermocouple was attached with a sphere at different locations (Bottom, Side, and Top) then dipped it inside the Styrofoam box which was filled with liquid nitrogen. The estimated time for different spheres to reach the cryogenic temperature ($192{ }^{\circ} \mathrm{C}$ ) is calculated with the help of lumped parameter analysis. The time is taken by the sphere of $4.14 \mathrm{~cm} 3.18 \mathrm{~cm}$ and $2.70 \mathrm{~cm}$ diameters to reach the steady state temperature of $-192{ }^{\circ} \mathrm{C}$ were five minutes forty-six seconds, four minutes twenty seconds and three-minute forty-nine seconds respectively.

$$
\left(T-T_{\infty}\right) /\left(T_{i}-T_{\infty}\right)=\exp ^{(-h A t / \rho v c)}
$$

Steady-state temperature is also measured with the help of thermocouple. Boiling of liquid nitrogen is stopped when steady-state temperature of the sphere was reached. It was the practical indication that the sphere could achieve the desired temperature. After that, the chilled sphere is removed from liquid nitrogen and dipped in different concentrations of brine solution.

The brine solution had been prepared by using Tata salt. The concentration of brine solution was prepared with the weight of solute per unit weight of solvent. It means, for $5 \%$ brine solution, One-kilogram Tata salt will be dissolved in nineteen kilograms of water. Similarly, for $10 \%$ brine solution, two kilograms of salt is dissolved in eighteen kilograms of water and for $15 \%$ brine solution; three kilograms of salt will be dissolved in seventeen kilograms of water. When the surface of chilled sphere touched the brine solution, ice is formed on the outer surface of the sphere within a fraction of seconds. After two to three seconds of the initial contact of the cooled sphere with the solution, the rate of formation of ice is reduced due to initial ice layer acts as an obstruction and having lower thermal conductivity with respect to that of stainless steel. The temperature of a sphere is reduced due to absorbing the latent heat from the solution during the solidification process. The decrement in temperature is measured with respect to time for different spheres with the help of DAQ system. The thickness of ice formation on the outer surface of a sphere is measured manually with the help of steel scale for different time 
intervals. Vernier caliper was not suitable for this because ice melts quickly when it came in contact with Vernier caliper or by applying some pressure. Three concentration of brine solution was used (5-15\%). When the temperature of brine solution was below its freezing temperature, pure water solidifies into ice and salt crystals are separated from it. The movement of phase front was depended on the initial concentration of salt in water, which was responsible for variation in thickness of ice formed. When the concentration of the solution was increased, then the rate of thickness of ice layer will be decreased and vice versa, this phenomenon satisfied the result of Matsumoto et al. [5]. Natural convection occurred inside the brine solution due to heat transfer from brine solution to the solid sphere. To reduce the effect of forced convection, we placed the sphere inside the brine solution carefully. The brine solution came in contact with cool sphere loses its latent heat and get denser. The dense solution moved downward due to gravity and this phenomenon is continued until the melting takes place. Due to density, gradient occurred in the contacting zone the thickness of ice was maximum at the bottom surface. As we moved from bottom to top surface, the ice thickness was decreasing. Upper half section ice formation was almost symmetrical whereas lower half was unsymmetrical due to density gradient.

\subsection{Ice thickness for different concentration, at different time interval and at different locations}

To measure the thickness of ice formed on the outer surface of different spheres, steel scale, and small hammer are used. Ice thickness is measured for different time intervals and for different concentration of brine solution at the top, side and bottom surfaces respectively as shown in Table 1.

Table 1. Thickness of ice at different time interval for different concentration

\begin{tabular}{|c|c|c|c|c|c|}
\hline $\begin{array}{c}\text { Concentration } \\
(\%) \\
\end{array}$ & $\begin{array}{c}\text { Diameter } \\
\text { (cm) }\end{array}$ & $\begin{array}{l}\text { Time } \\
\text { (s) }\end{array}$ & $\begin{array}{c}\text { Top } \\
(\mathbf{m m})\end{array}$ & $\begin{array}{l}\text { Side } \\
(\mathrm{mm})\end{array}$ & $\begin{array}{c}\text { Bottom } \\
(\mathrm{mm})\end{array}$ \\
\hline 5 & 4.14 & 60 & 2.75 & 4.00 & 5.25 \\
\hline 5 & 4.14 & 90 & 3.00 & 4.50 & 5.50 \\
\hline 5 & 4.14 & 120 & 2.50 & 4.00 & 5.80 \\
\hline 5 & 3.18 & 60 & 2.00 & 3.25 & 4.50 \\
\hline 5 & 3.18 & 90 & 2.00 & 3.00 & 4.50 \\
\hline 5 & 3.18 & 120 & 1.00 & 2.00 & 4.00 \\
\hline 5 & 2.70 & 60 & 1.50 & 2.50 & 3.50 \\
\hline 5 & 2.70 & 90 & 0.00 & 1.00 & 2.50 \\
\hline 5 & 2.70 & 120 & 0.00 & 0.00 & 2.00 \\
\hline 10 & 4.14 & 60 & 2.25 & 3.50 & 4.50 \\
\hline 10 & 4.14 & 90 & 2.00 & 3.00 & 4.80 \\
\hline 10 & 4.14 & 120 & 0.80 & 2.50 & 4.00 \\
\hline 10 & 3.18 & 60 & 2.00 & 2.50 & 3.00 \\
\hline 10 & 3.18 & 90 & 1.50 & 2.00 & 3.00 \\
\hline 10 & 3.18 & 120 & 0.00 & 0.00 & 0.00 \\
\hline 10 & 2.70 & 60 & 1.50 & 1.80 & 2.75 \\
\hline 10 & 2.70 & 90 & 0.00 & 0.00 & 2.00 \\
\hline 10 & 2.70 & 120 & 0.00 & 0.00 & 0.00 \\
\hline 15 & 4.14 & 45 & 1.20 & 2.00 & 3.00 \\
\hline 15 & 4.14 & 60 & 0.90 & 2.00 & 3.00 \\
\hline 15 & 4.14 & 90 & 0.60 & 1.00 & 3.00 \\
\hline 15 & 4.14 & 120 & 0.00 & 0.00 & 2.00 \\
\hline 15 & 3.18 & 45 & 0.80 & 1.50 & 2.00 \\
\hline 15 & 3.18 & 60 & 0.70 & 1.00 & 1.50 \\
\hline 15 & 3.18 & 90 & 0.00 & 0.00 & 1.00 \\
\hline 15 & 2.70 & 45 & 0.50 & 1.00 & 2.00 \\
\hline 15 & 2.70 & 60 & 0.00 & 0.50 & 1.00 \\
\hline
\end{tabular}

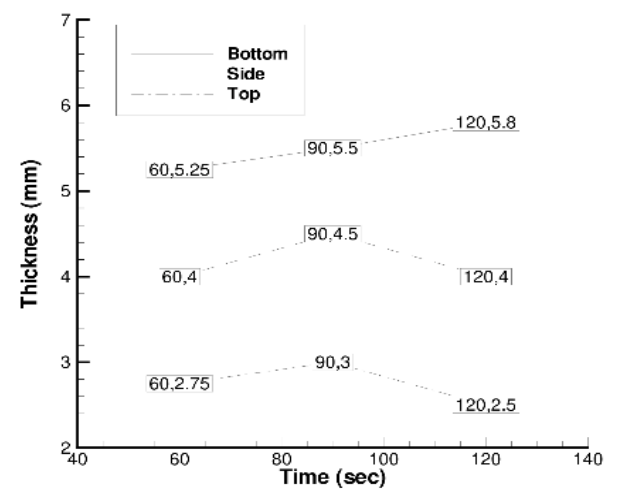

(a)

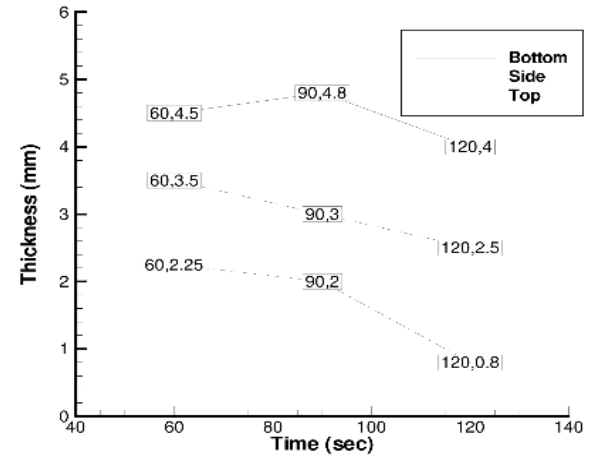

(b) 


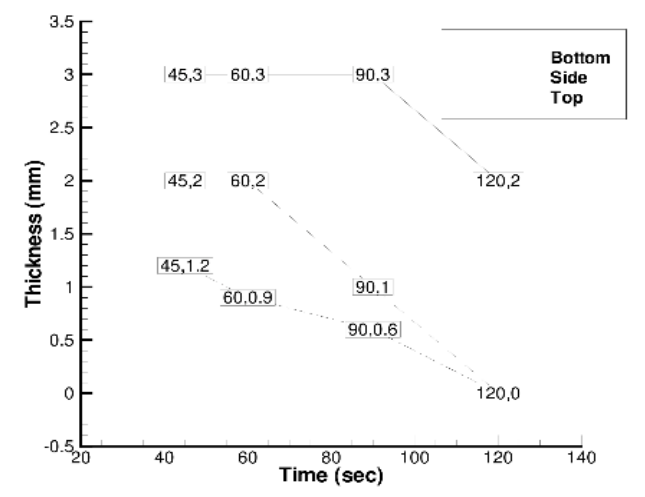

(c)

Figure 2. Variation of thickness with respect to time for 4.14 $\mathrm{cm}$ diameter sphere (a) $5 \%$ brine solution (b) $10 \%$ brine solution (c) $15 \%$ brine solution

The time is counted when the bottom of the cool sphere had touched the brine solution with the help of stopwatch. After a definite time-period, the sphere is removed from the solution. It is broken for ice thickness measurement. The process is repeated for different concentrations and time intervals. Figure 2 represents the thickness of ice for $4.14 \mathrm{~cm}$ diameter sphere was maximum in $5 \%$ solution $(5.8 \mathrm{~mm}$ at the bottom surface) after 120 seconds whereas at the side and top surfaces $(4.5 \mathrm{~mm}$ and $3 \mathrm{~mm}$ ) after 90 seconds. On the other hand, for $10 \%$ solution, maximum ice thickness at the bottom surface occurred after 90 seconds $(4.8 \mathrm{~mm})$ whereas at the side and top surfaces $(3.5 \mathrm{~mm}$ and $2.25 \mathrm{~mm})$ after 60 seconds. For this specification, melting of ice at the top surface was too fast after 90 seconds. For $15 \%$ solution, the bottom surface had constant ice thickness $(3 \mathrm{~mm})$ up to 90 seconds then melting started. Similarly, side surface had constant ice thickness $(2 \mathrm{~mm})$ up to 60 seconds. At the top surface, continuous decreasing trend occurred after 45 seconds.

Figure 3 represents the thickness of ice for $3.18 \mathrm{~cm}$ diameter sphere, maximum ice thickness for $5 \%$ solution obtained at the bottom after 90 seconds $(4.5 \mathrm{~mm})$ whereas at side and bottom after 60 seconds ( $3.25 \mathrm{~mm}$ and $2 \mathrm{~mm}$ ). When the concentration was increased to $10 \%$ then thickness was constant up to 90 seconds $(3 \mathrm{~mm})$ at the bottom whereas side and top surfaces had continuous decreasing trend after 60 seconds. For $15 \%$ solution, maximum thickness obtained at all the locations after 45 seconds thereafter ice thickness was continuously decreasing.

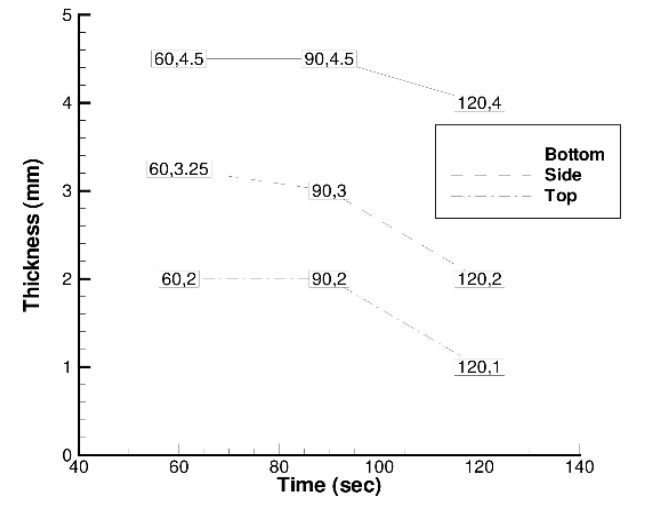

(a)

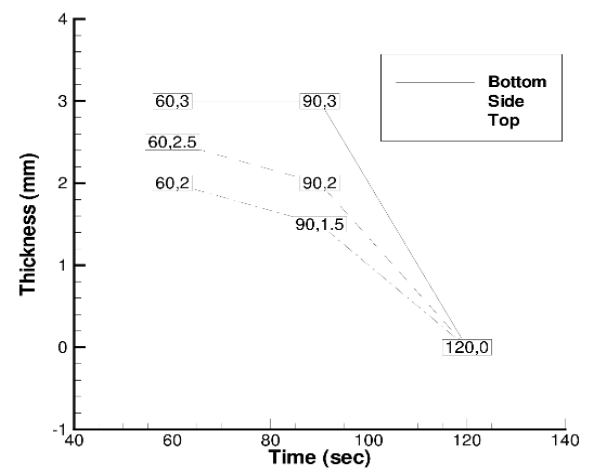

(b)

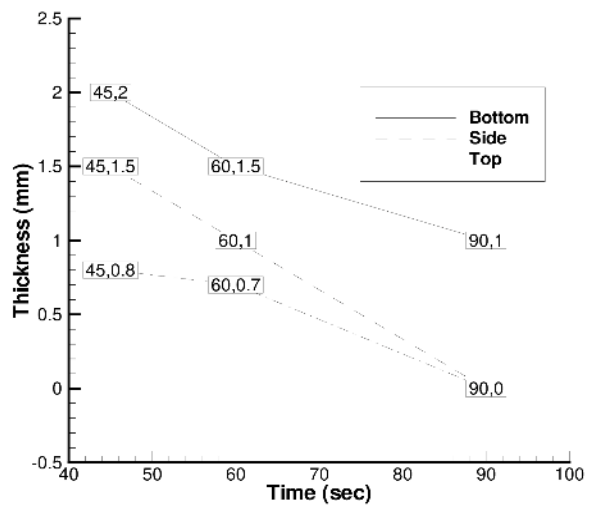

(c)

Figure 3. Variation of thickness with respect to time for 3.18 $\mathrm{cm}$ diameter sphere (a) $5 \%$ brine solution (b) $10 \%$ brine solution (c) $15 \%$ brine solution

Figure 4 represents the thickness of ice for $2.72 \mathrm{~cm}$ diameter sphere, maximum thickness obtained after 60 seconds $(3.5 \mathrm{~mm}$, $2.5 \mathrm{~mm}, 1.5 \mathrm{~mm}$ at the bottom, side and top surfaces respectively) thereafter-continuous decreasing trend occurs for $5 \%$ solution. If the concentration is increased to $10 \%$, maximum thickness obtained after 60 seconds, which was less as compared to previous one. For this specification, the thickness of ice at the side and top surfaces have less difference but their melting pattern was similar. For $15 \%$ solution, maximum ice thickness obtained after 45 seconds thereafter continuous melting occurred at all the surfaces.

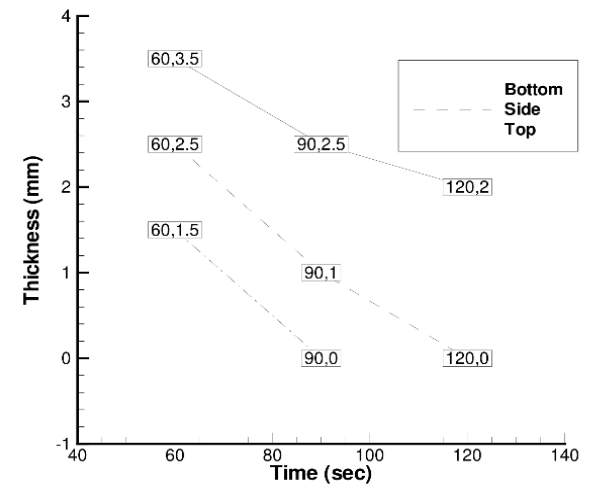

(a) 


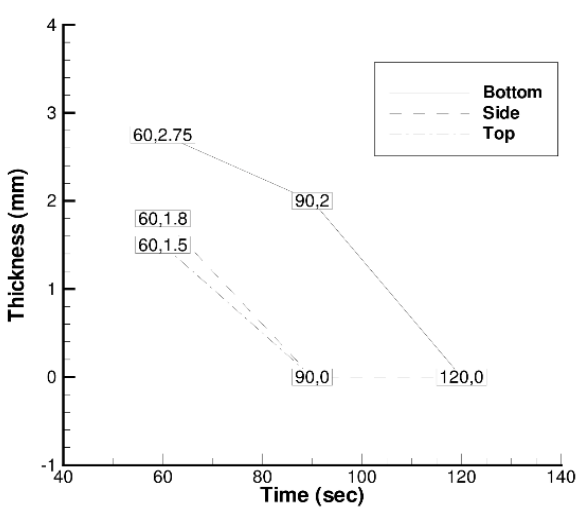

(b)

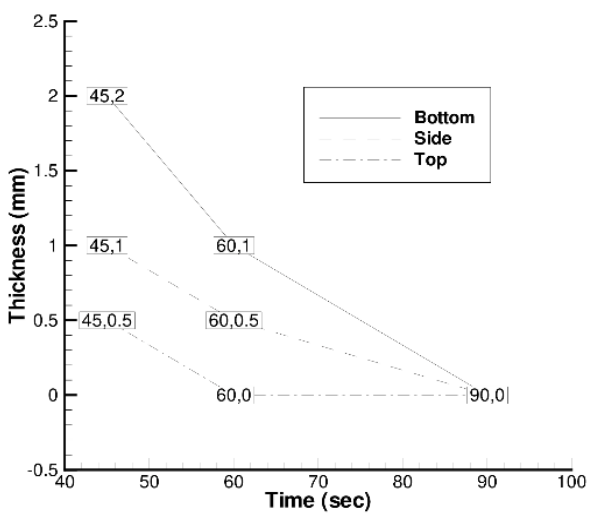

(c)

Figure 4. Variation of thickness with respect to time for 2.72 $\mathrm{cm}$ diameter sphere (a) $5 \%$ brine solution (b) $10 \%$ brine solution (c) $15 \%$ brine solution

\section{RESULT AND DISCUSSION}

To study the effect of concentration on the thickness of ice for different time intervals, experiments were performed and data are shown as in the Table 1 . For $5 \%$ concentration brine solution up to 60 seconds, ice was not melted for all the spheres. Ice quality was soft compared to that made when immersed into pure water and the melting pattern of ice happens in a different way. As time duration is increased to 90 seconds then Ice melted at top and side surfaces for $2.70 \mathrm{~cm}$ diameter sphere only which is shown in Figure 5.

Now, further increase the time duration to 120 seconds, for $4.14 \mathrm{~cm}$ diameter sphere, the ice melted from the top surface only.

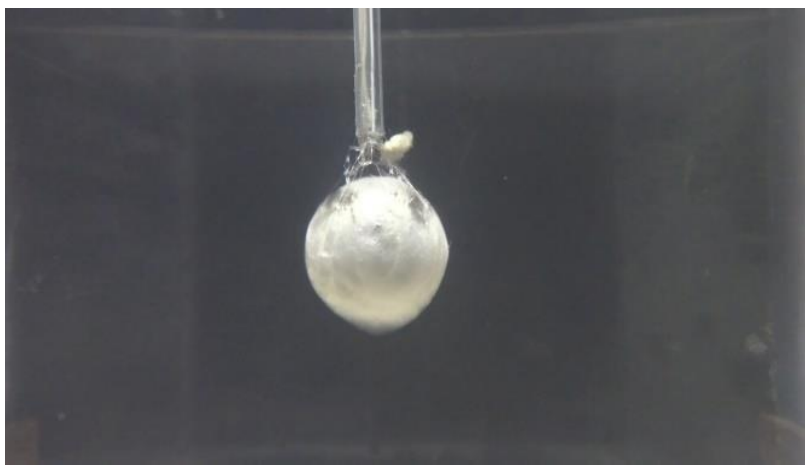

(a)

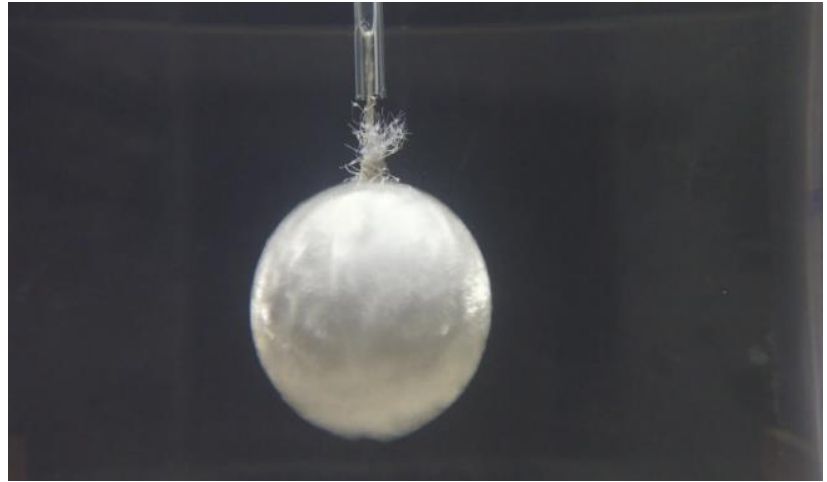

(b)

Figure 5. (a) Melting of ice for $2.70 \mathrm{~cm}$ sphere and $5 \%$ concentration brine solution (b) $4.14 \mathrm{~cm}$ diameter sphere after 120 seconds in $5 \%$ concentration brine solution

For $3.18 \mathrm{~cm}$ sphere, ice lost its strength and after ten seconds melting started from top and side surfaces. For $2.70 \mathrm{~cm}$ sphere, the ice melted from top and side surfaces and at the bottom, mushy ice is available which is 1.5 to $2 \mathrm{~mm}$ in thickness. The melting pattern is shown in the Figure 6.

Ice quality was relatively good but strength was less as compared to other time states due to melting started in this time interval for all the spheres. When the concentration is changed to $10 \%$, the total time (solidification as well as melting) would be reduced as compared to the previous one because of salt concentration increased the solidification rate by decreasing the phase change temperature of water present in the solution whereas, during melting, rate of melting of ice was also increased.

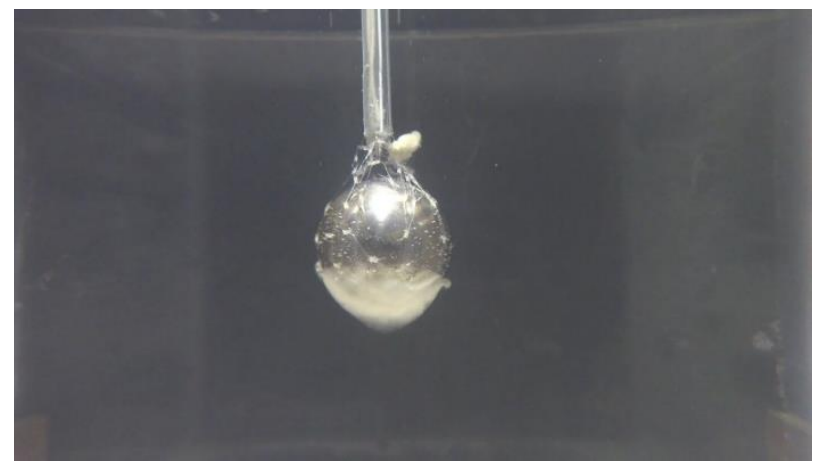

Figure 6. $2.70 \mathrm{~cm}$ diameter sphere after 120 seconds in 5\% concentration brine solution

In this solution for a 60 -second time duration, Ice had good strength for $4.14 \mathrm{~cm}$ diameter whereas for $3.18 \mathrm{~cm}$ diameter ice quality is soft whereas, for $2.70 \mathrm{~cm}$ diameter, melting started from top surface that's why ice quality is relatively soft with respect to $3.18 \mathrm{~cm}$ sphere. This might be happened due to decrease in total time after increasing the concentration of brine solution. When hammering the ice ball for measurement of thickness, it fractured, so thickness will be measured with care and break the ice pieces by applying small force. This phenomenon is not obtained in $5 \%$ concentration solution. The important observation in this concentration is melting phenomenon of the ice. The ice melted in a different manner with respect to previous concentration. At the top half of the ice ball, the ice melted like just leaving the surface of sphere whereas that of bottom half melted in a sequential manner like 
unwrapping of thread on the surface of a sphere, which is shown in the Figure 7.

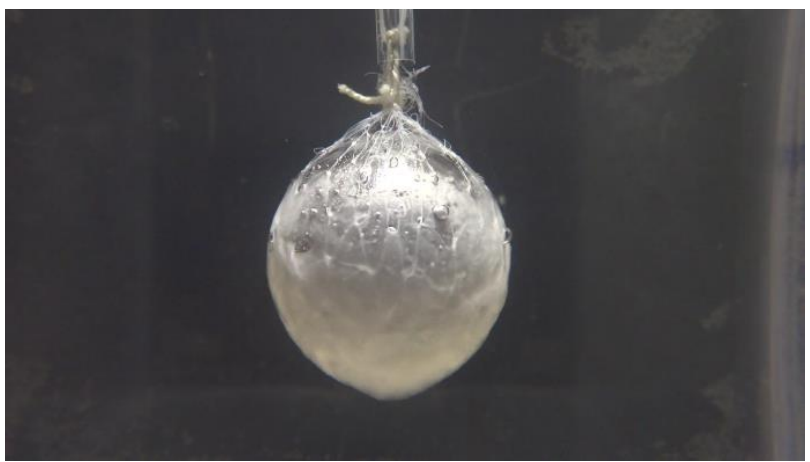

(a)

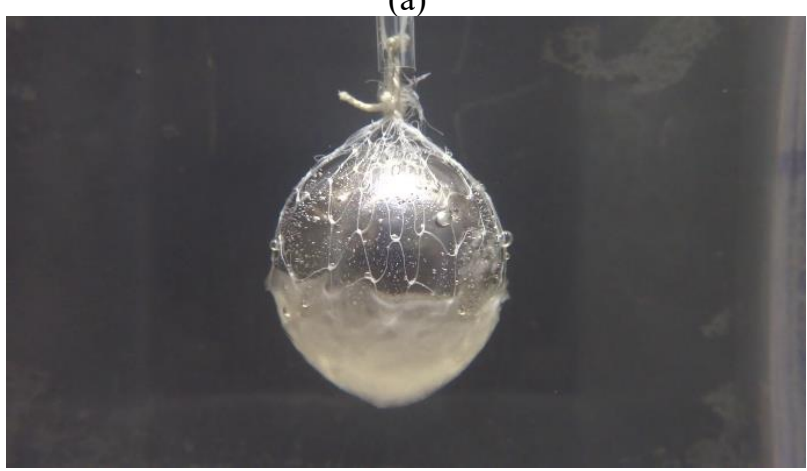

(b)

Figure 7. Comparison of melting of ice in $10 \%$ brine solution at upper and lower section at different time

For 90 seconds time duration, Ice strength was good but fracture after hammer. For $3.18 \mathrm{~cm}$ steel ball, melting started after 65 seconds from the top surface. Ice quality was relatively soft for this specification as compared to that of 4.14 $\mathrm{cm}$ sphere. For $2.70 \mathrm{~cm}$ diameter sphere, top and side surface melted completely. Melting started after 62 seconds. Mushy ice remained at the bottom due to unwrapping behavior of melting but was not measurable. Now, further study for 120 seconds, for $4.14 \mathrm{~cm}$ diameter sphere, melting started from the top surface after 96 seconds. For $3.18 \mathrm{~cm}$ diameter sphere, top and side surfaces have been melted completely. Only mushy ice remained at the bottom surface, which was $2 \mathrm{~mm}$ in thickness. The ice starts melting after 62 seconds for that specification. For $2.70 \mathrm{~cm}$ ice ball, melting started from the top surface after 45 seconds. No ice remained on any surface due to decrease in total time. For $15 \%$ concentration brine solution, total duration (solidification and melting) is reduced quite significantly. The important change occurred in this concentration, which was the melting process. The ice particles, in the process of melting moved upward due to the density difference between brine solution and ice particles.

This phenomenon happened slightly in $10 \%$ solutions but completely absent in $5 \%$ brine solution. Since the total time is reduced hence for this concentration, measurement of ice thickness started after 45 seconds. For this time duration, no melting occurred at any surface. Only solidification and melting time would be decreased as compared to previous concentrations. When time duration is increased to 60 seconds, then for $4.14 \mathrm{~cm}$ diameter ice ball, melting does not happen. Ice thickness on the outer surface is relatively decreased but strength was good. For $3.18 \mathrm{~cm}$ diameter sphere, melting started from top the surface only. For $2.70 \mathrm{~cm}$ diameter ice ball, melting started after 85 seconds. Top surface has no ice whereas at the side surface, very thin layer which is not measurable. Only bottom ice thickness is measured. Now further increase the time duration to 90 seconds, for $4.14 \mathrm{~cm}$ diameter sphere, melting started from top and side surfaces. For $3.18 \mathrm{~cm}$ diameter sphere, no ice layer remained at top and side surface, only soft ice layer (mushy ice) remained at the bottom surface whereas, for $2.72 \mathrm{~cm}$ sphere, the ice melted completely. For 120 seconds, no ice remains at the top and side surfaces, only soft ice layer (mushy ice) remained at the bottom surface for $4.14 \mathrm{~cm}$ diameter sphere.

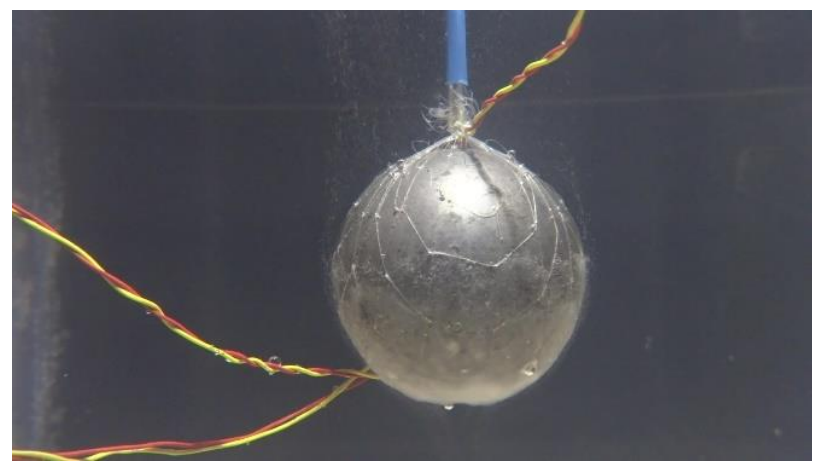

(a)

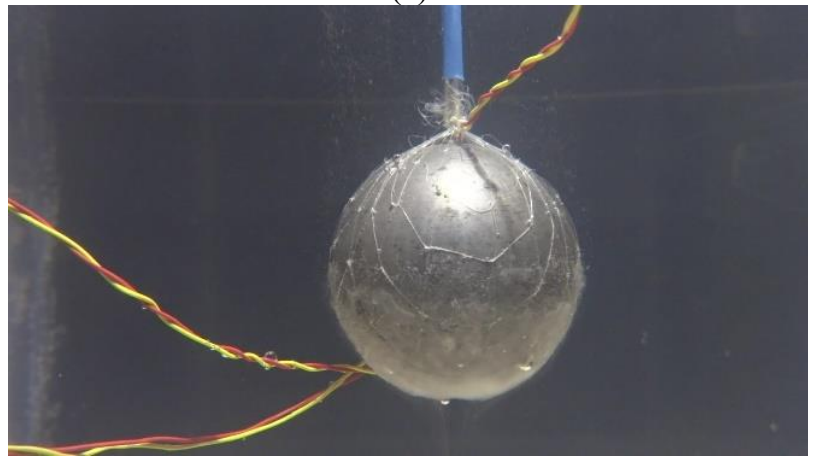

(b)

Figure 8. Melting of ice in $15 \%$ brine solution

\subsection{Temperature variation at top, side and bottom surfaces of cooled sphere inside different concentration of brine solution}

The sphere is dipped in the liquid nitrogen. After a specified time-period, the steady state temperature is reached $\left(-192^{\circ} \mathrm{C}\right)$, the chilled sphere was dipped inside the brine solution. The temperature of bottom surface is drastically reduced to $-23{ }^{\circ} \mathrm{C}$ due to direct contact of thermocouple bid to brine solution whereas temperature of side surface is reduced to $-11{ }^{\circ} \mathrm{C}$ but top surface temperature reduces slowly and takes four to five seconds to reach $-89^{\circ} \mathrm{C}$. Since, temperature of the sphere was too low, ice formation takes place within fraction of seconds. When the very first enclosure of ice layer is formed thereafter ice thickness increases slowly because of thermal conductivity of ice was very low as compared to that of steel. After 125 seconds, the temperature at the bottom, side, and top surfaces are $-10{ }^{\circ} \mathrm{C}, 0{ }^{\circ} \mathrm{C}$ and $0{ }^{\circ} \mathrm{C}$ respectively for $4.14 \mathrm{~cm}$ diameter sphere which is shown in Figure 9.

During immersion of the chilled sphere inside brine solution, air particles are trapped in the gap between the sphere and high-density polyethylene net. These air particles had no time to remove during solidification of ice but during melting, it came out and bubbles are formed which is shown in Figure 10.

The point at which bubbles are formed, temperature varied 
frequently. For $3.72 \mathrm{~cm}$ diameter sphere After eighty-five seconds, the temperature at the bottom, side, and top surfaces are $-19{ }^{\circ} \mathrm{C}, 0{ }^{\circ} \mathrm{C}$ and $0{ }^{\circ} \mathrm{C}$ respectively. For $2.70 \mathrm{~cm}$ diameter sphere after seventy-three seconds, the temperature at the bottom, side, and top surfaces are $-7{ }^{\circ} \mathrm{C},-3.5^{\circ} \mathrm{C}$ and $0{ }^{\circ} \mathrm{C}$ respectively shown in Figure 11-12.

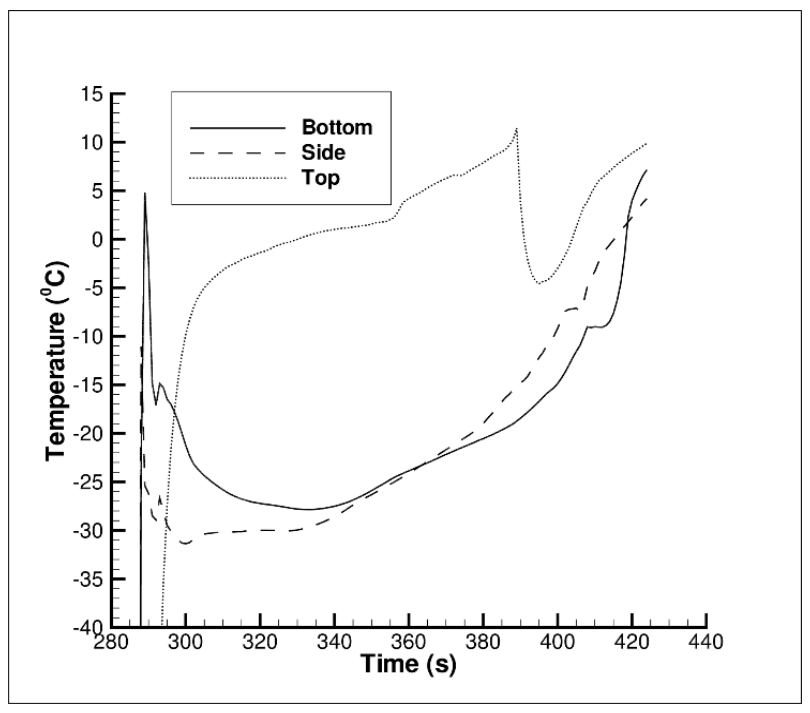

Figure 9. Temperature Vs Time plot at different surfaces for $4.14 \mathrm{~cm}$ solid sphere inside $15 \%$ brine solution

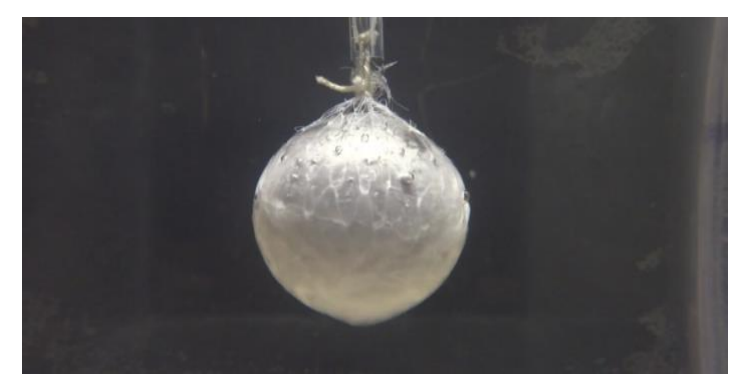

Figure 10. Air bubbles during melting of ice

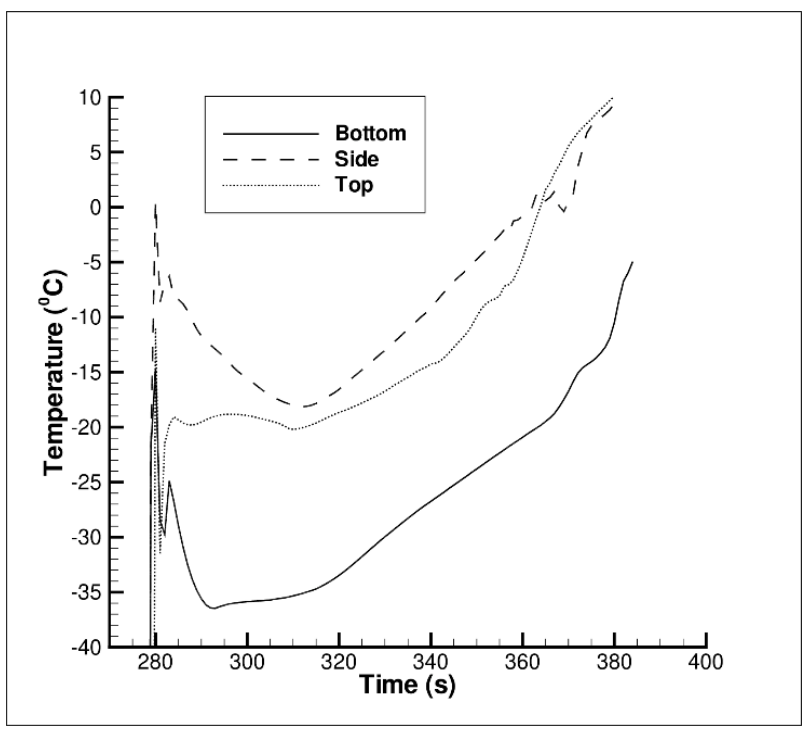

Figure 11. Temperature Vs Time plot at different surfaces for $3.18 \mathrm{~cm}$ solid sphere inside $15 \%$ brine solution

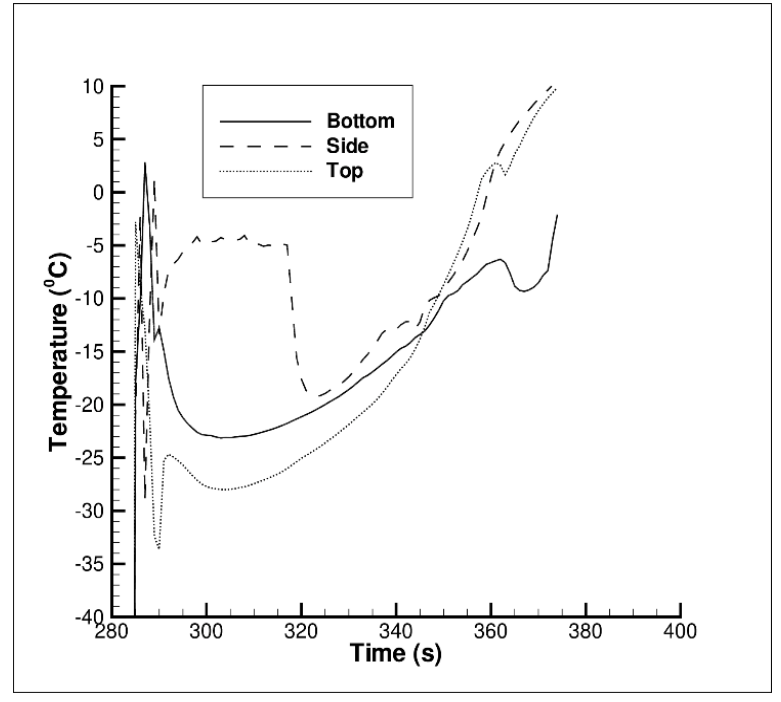

Figure 12. Temperature Vs Time plot at different surfaces for $2.70 \mathrm{~cm}$ solid sphere inside $15 \%$ brine solution

\section{CONCLUSION}

The experiment is performed to visualize the solidification and melting behavior of stainless steel when it was chilled up to $-192{ }^{\circ} \mathrm{C}$ then immersed in brine solution of different concentrations. The thickness of ice formation and temperature variation with respect to time is also measured. As the concentration of brine solution was increased, the thickness of ice formation and total time (solidification and melting) is decreased. When the concentration of brine solution increased the strength of ice is increased but it is difficult to break after hammering. Due to increase in concentration mushy region shifted below $0{ }^{\circ} \mathrm{C}$, it is the main reason for the reduction in total time. Extreme corrosiveness would be the major disadvantage of $\mathrm{NaCl}$ water brine solution. The experimental result obtained here might be used in geological and industrial projects wherever brine solution appeared. Since the chilled sphere is dipped steadily into the brine solution hence no forced convection occurred. Heat transfer happened only through natural convection but it could not visualize due to very low convection current. Melting is started from the top surface of the sphere for each concentration and its pattern was different for different concentrations. Four assumptions made for the experiment (a) Property of material such as thermal conductivity; density, surface roughness etc. are not changed when it was dipped in the liquid nitrogen. (b) Sphere dimension was not changed. (c) Ice formation took place from the surface of the sphere. (d) The thickness of high-density polyethylene net is assumed negligible and it did not obstruct the heat transfer rate. (e) The sphere was assumed to be lump to calculate the estimated time to reach at $-192{ }^{\circ} \mathrm{C}$ when it was dipped in the liquid nitrogen. To capture the clear video and picture, we use Sony cyber-shot DSC-H300 point and shoot camera.

\section{REFERENCES}

[1] Matin MH, Pop I, Khanchezar S. (2013). Natural convection of power-law fluid between two square Eccentric duct annuli. Journal of Non-Newtonian Fluid 
Mechanics

197:

$11-23$.

https://doi.org/10.1016/j.jnnfm.2013.02.002

[2] Christension MS, Incropera FP. (1988). Solidification of an aqueous ammonium chloride solution in a rectangular cavity-1 experimental study. International Journal of Heat and Mass Transfer 32: 47-68. https://doi.org/10.1016/0017-9310(89)90090-2

[3] Bell MA, Smith IE. (1980). Thermal energy storage using saturated salt solution. Peragon press Limited 5: 1085-1090. https://doi.org/10.1016/0360 5442(80)90031-6

[4] Choi J, Viskanta R. (1992). Freezing of aqueous sodium chloride solution saturated packed bed from a vertical wall of a rectangular cavity. International Journal of Heat and Mass Transfer 36: 2805-2813. https://doi.org/10.1016/0017-9310(93)90100-K

[5] Matsumoto K, Okada M, Murakami M, Yabushita Y. (1995). Solidification of porous medium saturated with aqueous solution in a rectangular cell ii. International Journal of Heat and Mass Transfer 38: 2935-2943. https://doi.org/10.1016/0017-9310(93)90106-g

[6] Okawa S, Saito A, Minami R. (2001). The solidifi cation phenomenon of the super cooled Water containing solid particles. International Journal of Refrigeration 24: 108117. https://doi.org/10.1016/S0140-7007(00)00060-8

[7] Vrbka L, Jungworth P. (2005). Brine rejection from freezing salt solutions a molecular dynamics Study. Physical Review Letters 95: 148501.

[8] Lucas T, Chourot JM, Bohuon PH, Flick D. (2001). Freezing of a porous medium in contact with a Concentrated aqueous freezant-numerical modelling of coupled heat and mass transport. International Journal of Heat and Mass Transfer 44: 2093-2106. https://doi.org/10.1016/s0017-9310(00)00238-6

[9] Valenza JJ, Scherer GW. (2007). A review of salt scaling mechanisms. Cement and Concrete Research 37: 10221034. https://doi.org/10.1016/j.cemconres.2007.03.005

[10] Wilson PW, Haymet ADJ. (2010). Effect of ice growth rate on the measured workman Reynolds freezing potential between ice and dilute $\mathrm{NaCl}$ solutions. The Journal of Physical Chemistry 114: 12585-12588. https://doi.org/10.1021/jp105001c

[11] Frederking THK, Clark JA. (1963). Natural convection film boiling over a sphere. Advances in Cryogenics Engineering 8: 501-506.

[12] Daney DE. (1975). Turbulent natural convection of liquid deuterium, liquid hydrogen \& nitrogen Within enclosed vessel. International Journal of Heat And Mass Transfer 19: 431-441. https://doi.org/10.1016/00179310(76)90099-5

[13] Hilal MA, Boom RW. (1979). An experimental study of free conv. heat transfer in supercritical helium. International Journal of Heat and Mass Transfer 67: 2235-2246.

[14] Merkin JH. (1977). Mixed convection from a horizontal circular cylinder. International Journal of Heat and Mass Transfer 45: 877-889. https://doi.org/10.1016/00179310(77)90086-2

[15] Bang KH. (1994). Forced convection heat transfer film boiling over a sphere for vapor and liquid flows. International Journal of Heat and Mass Transfer 37: 2415-2424. 9310(94)90283-6

[16] Rahli O, Topin F, Tadrist L, Pantaloni J. (1996). Analysis of heat transfer with liquid-vapor phase change in a forced ow fluid moving in a porous media. International Journal of Heat and Mass Transfer 39: 3959-3975. https://doi.org/10.1016/0017-9310(96)00053-1

[17] Kolev NI. (1998). Film boiling on vertical plates and spheres. Experimental Thermal and Fluid Science 18: $97-$ 115. https://doi.org/10.1016/s0894-1777(98)10021-3

[18] Miller B, Merriman B. (1999). Vapor-liquid interfacial dynamics and related liquid nitrogen. Cryogenics 39: 389-397. https://doi.org/10.1016/s0011-2275(99)000429

[19] Ettouney HM, Alatiqi I, Al-Sahali M, Al-Ali SA. (2004). Heat transfer Enhancement by metal screens and metal spheres in phase change energy storage systems. Renewable Energy 29: 841-860. https://doi.org/10.1016/j.renene.2003.11.003

[20] Koizumi H. (2004). Time and spatial heat transfer performance around an isothermally heated sphere Placed in a uniform, downwardly directed ow (in relation to the enhancement of latent heat storage Rate in a spherical capsule). Applied Thermal Engineering 24: 2583-2600.

https://doi.org/10.1016/j.applthermaleng.2004.03.011

[21] Benmansour A. (2006). Experimental and numerical investigation of solid particles thermal energy storage Unit. International Journal of Heat and Mass Transfer 59: 853-858.

https://doi.org/10.1016/j.applthermaleng.2005.07.014

[22] Tan FL. (2008). Constrained and unconstrained melting inside a sphere. International Communications in Heat and Mass Transfer 35: 466-475. https://doi.org/10.1016/j.icheatmasstransfer.2007.09.00 8

[23] Tan FL, Hosseinizadeh SF, Khodadadi JM, Fan L. (2009). Experimental and computational study of constrained melting of phase change materials (PCM) inside a spherical capsule. International Journal of Heat and Mass Transfer 52: 3464-3472. https://doi.org/10.1016/j.ijheatmasstransfer.2009.02.043

[24] Jouharaa H, Axcell BP. (2009). Film boiling heat transfer and vapour film collapse on spheres. International Journal of Heat and Mass Transfer 56: 1137-1148. https://doi.org/10.1016/j.nucengdes.2009.04.008

[25] Kousksou T, El Rhaki T, El Omari K, Zeraouli Y, Le Guer Y. (2010). Forced convective heat transfer in supercooled phase-change material suspensions with stochastic crystallization. International Journal of Refrigeration 33: 1569-1582. https://doi.org/10.1016/j.ijrefrig.2010.05.006

[26] Kasim ARM. (2012). Solid sphere with natural convection boundary layer flow. International Journal of Thermal Sciences 1: 522-534.

[27] Hosseinizadeh SF, Tan FL, Darzi AAR. (2012). Numerical investigations of unconstrained melting of nano-enhanced phase change material (NEPCM) inside a spherical container. International Journal of Thermal Sciences 51: 77-83. https://doi.org/10.1016/j.ijthermalsci.2011.08.006

[28] Matin MH, Pop I, Khanchezar S. (2013). Natural convection of power-law fluid between two square Eccentric duct annuli. Journal of Non-Newtonian Fluid Mechanics 197: 11-23. https://doi.org/10.1016/j.jnnfm.2013.02.002

[29] Tham L, Nazar R. (2012). Mixed convection ow about a 
solid sphere embedded in a porous medium filled with Nano-fluid. Sains Malaysiana 41(12): 1643-1649.

[30] Oro E, Gracia A, Castell A, Farid MM, Cabeza LF. (2012). Phase change materials for cold thermal energy storage applications. Applied Energy 99: 513-533.

[31] Zhao W, Neti S, Oztekin A. (2013). Heat transfer analysis of encapsulated phase change Material for thermal energy storage. Applied Thermal Engineering 50: 143-151.

https://doi.org/10.1016/j.ijheatmasstransfer.2013.03.061

[32] Kiani H, Sun D, Zhang Z. (2013). Effects of processing parameters on the convective heat transfer rate during ultrasound assisted low temperature immersion treatment of a stationary sphere. Journal of Food Engineering 115: 384-390. https://doi.org/10.1016/j.jfoodeng.2012.10.029

[33] Hashemi Z, Abouali O, Kamali R. (2014). Threedimensional thermal heating cooling spheres falling in a newtonian liquid. International Journal of Thermal Sciences 82:

23-33. https://doi.org/10.1016/j.ijthermalsci.2014.03.008

[34] Amin NAM, Bruno F, Belusko M. (2014). Effective thermal conductivity for melting in PCM encapsulated in a sphere. Applied Energy 122: 280-287. https://doi.org/10.1016/j.apenergy.2014.01.073

\section{NOMENCLATURE}

$\mathrm{T}$

$\mathrm{T} \infty$

$\mathrm{Ti}$

$\mathrm{h}$

A

$\mathrm{t}$

$\mathrm{c}$

$\mathrm{Gr}$

$\operatorname{Pr}$

$\mathrm{Nu}$

$\mathrm{Ra}$

$\operatorname{Re}$

Ste

$\mathrm{Cp}$

\section{Greek symbols}

$\rho$
$\mu$
$\beta$ temperature achieved by the solid sphere when it was dipped in the liquid nitrogen temperature of liquid nitrogen

temperature of brine solution convective heat transfer coefficient of liquid nitrogen

surface area of sphere

time taken by the sphere to achieve steady state temperature volume of sphere

specific heat capacity of stainless steel

Grashof number

Prandtl number

Nusselt number

Rayleigh number

Reynold number

Stefan number

Specific heat capacity

density of stainless steel absolute viscosity

coefficient of thermal expansion 